
\title{
25 Research Soure \\ Knockdown of hspg2 is associated with mandibular jaw joint fusion and neural crest cell dysfunction in zebrafish
}

\section{Barbara S. Castellanos}

University of Texas at El Paso

Anita Quintana ( $\sim$ aquintana8@utep.edu)

University of Texas at El Paso https://orcid.org/0000-0002-3596-1587

\section{Research article}

Keywords: HSPG2, neural crest cells, craniofacial development

Posted Date: June 3rd, 2020

DOI: https://doi.org/10.21203/rs.3.rs-27670/v1

License: (c) (1) This work is licensed under a Creative Commons Attribution 4.0 International License.

Read Full License

Version of Record: A version of this preprint was published on March 8th, 2021. See the published version at https://doi.org/10.1186/s12861-021-00238-4. 


\section{Abstract}

Background. Heparan sulfate proteoglycan 2 (HSPG2) encodes for perlecan, a large proteoglycan that plays an important role in cartilage formation, cell adhesion, and basement membrane stability. Mutations in HSPG2 have been associated with Schwartz-Jampel syndrome and Dyssegmental Dysplasia Silverman-Handmaker Type, two disorders characterized by skeletal abnormalities. These data indicate a function for HSPG2 in cartilage development/maintenance. However, the mechanisms in which HSPG2 regulates cartilage development are not completely understood. Here, we explored the relationship between this gene and craniofacial development through morpholino-mediated knockdown of $h s p g 2$ in zebrafish.

Results. Knockdown of $h s p g 2$ resulted in a fusion of the mandibular jaw joint at 5 days post fertilization (dpf). We surmised that defects in mandible development were a consequence of neural crest cell (NCC) dysfunction, as these multipotent progenitors produce the cartilage of the head. Early NCC development was normal in morphant animals as measured by distal-less homeobox 2a ( $d / \times 2 a)$ and SRY-box transcription factor 10 (sox10) expression at $1 \mathrm{dpf}$. However, subsequent analysis at later stages of development (4 dpf) revealed a decrease in the number of Sox $10^{+}$and Collagen, type II, alpha $1 \mathrm{a}$ $(\mathrm{Col} 2 \mathrm{a} 1 \mathrm{a})^{+}$cells within the mandibular jaw joint region of morphants relative to random control injected embryos. Concurrently, morphants showed a decreased expression of NK3 homeobox 2 (nkx3.2), a jaw joint molecular marker at $4 \mathrm{dpf}$.

Conclusions. Collectively, these data suggest a complex role for hspg2 in jaw joint formation and late stage NCC differentiation.

\section{Background}

Heparan sulfate proteoglycan 2 (HSPG2) is a gene located on the human short arm $p$ of chromosome one at position 36.12 (1). HSPG2 encodes for a large, multidomain proteoglycan known as perlecan, which, despite its size and general complexity, is well conserved across various vertebrate species (1). After the process of glycosylation, perlecan is secreted into the basement membrane, a region rich with glycoproteins, collagen, and laminins that separates the epithelium from underlying connective tissue. Perlecan is also found in cartilage, which makes up most of the skeleton during early development $(1,2)$.

Previous studies have associated mutations in HSPG2 to Schwartz-Jampel Syndrome (SJS) and Dyssegmental Dysplasia Silverman-Handmaker Type (DDSH) (3-6). SJS is a rare disorder characterized by muscle stiffness (myotonia) and skeletal abnormalities (6). SJS is similar to DDSH, a less frequent but more severe autosomal recessive disorder characterized by similar musculoskeletal deformities (7). While skeletal abnormalities differ in severity from patient to patient, patterns emerge wherein most patients diagnosed with SJS or DDSH have craniofacial abnormalities specifically, with conditions like micrognathia (a small jaw with a receding chin) (8) manifesting in 30-79 percent of SJS patients and 80- 
99 percent of DDSH patients $(9,10)$. These manifestations of chondrodysplasia (disorders in the development of cartilage and later, bone) (11) suggest that HSPG2 regulates craniofacial development.

One manner in which this disruption could occur is through regulation of the neural crest cell (NCC) lineage. Previous studies have demonstrated that mutations in HSPG2 are associated with abnormal chondrocyte proliferation and arrangement (3). Chondrocytes are cells found in cartilage, which later undergoes the ossification process to become bone (12). In the face and neck, chondrocytes arise from NCCs, a multipotent progenitor cell population that forms at the dorsal end of the neural tube during neural tube closure. There are four populations of NCCs, but cranial NCCs (CNCCs) are those that develop into cartilage and bone, making them vital to proper craniofacial development (13-15). Even so, not much has been elucidated on the mechanisms that tie together how mutations in HSPG2 could contribute to chondrocyte disorganization and how this phenomenon manifests as craniofacial abnormalities.

To begin to understand these mechanisms, we performed morpholino mediated knockdown in the developing zebrafish to determine the cellular mechanisms by which $h s p g 2$ regulates craniofacial development. Knockdown of $h s p g 2$ caused mandibular jaw joint fusion and disrupted late stage differentiation of CNCCs, with little to no effect on early stage CNCC development. Collectively, our results suggest that $h s p g 2$ is essential for joint formation in the developing zebrafish.

\section{Results}

\section{Morpholino-induced knockdown of hspg2 is associated with craniofacial phenotypes}

Previous studies performed in the murine model showed that deletion of Hspg2 leads to failure of the chondro-osseous junction of developing bones; animals were also characterized by a narrow thorax and craniofacial abnormalities (3). Based on these studies, we hypothesized that morpholino mediated knockdown of the zebrafish hspg2 gene would cause craniofacial abnormalities. To test this, we performed alcian blue staining to detect developing cartilage. Measurements of the distance between the ceratohyal and Meckel's cartilage on the alcian blue stained embryos at 5 days-post-fertilization (dpf) (Figure 1A-D) showed that the injection of the translation blocking morpholino caused a $7 \%$ truncation in the zebrafish mandible when compared to the random control and non-injected wildtype groups. Stained embryos also demonstrated a fusion of the mandibular jaw joint between the Meckel's cartilage and palatoquadrate (the dorsal component of the mandibular arch) in the morphants, a phenotype not present in either the non-injected wildtype or random control groups (Figure $1 A^{\prime}-C^{\prime}$ ).

\section{nkx3.2 expression decreased in hspg2 morphants}

NK3 homeobox 2 ( $n k \times 3.2$ ) was first identified in the drosophila model and is part of the NK family of homeobox genes (16). Homologues of the gene have been found in vertebrates (including zebrafish and humans) and are dorsally expressed predominately in the first pharyngeal arch where the gene is essential for proper joint formation (16). Knockdown of $n k x 3.2$ causes mandibular jaw joint fusion in amphibians (16). Therefore, we utilized $n k \times 3.2$ expression as a marker of mandibular jaw joint 
development. In situ hybridization performed at $2 \mathrm{dpf}$ (Figure 2A-C) demonstrated decreased expression of $n k \times 3.2$ in the morphant groups when compared to the random control and non-injected wildtype groups. qPCR at $4 \mathrm{dpf}$ confirmed a statistically significant decrease in $n k x 3.2$ in morphants relative to the random control group (Figure 2D).

\section{Neural crest cells migrate normally in the absence of hspg2}

Because defects in the number of and migration of NCCs are possible mechanisms by which craniofacial deficits may arise (17), we hypothesized that the craniofacial abnormalities present at $5 \mathrm{dpf}$ might be due to early CNCC defects. To determine if $h s p g 2$ affects early CNCCs, we analyzed $T g$ (sox10: TagRFP) embryos at both the 18 somite (aligning with early NCC specification and migration) (18) and Prim-5 (corresponding with NCCs becoming situated in the pharyngeal arches) stages. Previous studies have used SRY-box transcription factor 10 (sox10) as a valid NCC marker during early developmental stages (17). Results revealed no discernable differences in the level of Sox $10^{+}$cells in $h s p g 2$ morphants and non-injected wildtype groups (Figure 3A-A' and 3B-B').

\section{dlx2a expression in morphants is unaffected}

Next we analyzed the expression of distal-less homeobox $2 \mathrm{a}(\mathrm{d} / \mathrm{x} 2 \mathrm{a})$ at the Prim-5 stage in random control and hspg 2 morpholino injected embryos to determine if CNCC specification occurs normally upon knockdown of $h s p g 2$. The expression of $d / x 2 a$ has been established as a marker of proper CNCC specification in previous studies $(19,20)$. In situ hybridization (Figure 4A-C) revealed that there was no significant difference in the expression of $d l \times 2 a$ in morphants relative to random control injected embryos. qPCR measurements performed at Prim- 5 in both groups validated the normal level of $d l \times 2 a$ expression in morphant animals (Figure 4D). Collectively, these data suggest that early CNCC development was normal.

\section{hspg2 knockdown affects cell numbers in jaw joint region}

We next hypothesized that $h s p g 2$ mediates late stage CNCC differentiation. To test this, we performed analysis of Sox $10^{+}$cells at 3 and 4 dpf using $\operatorname{Tg}$ (sox10:TagRFP) larvae. At 3 dpf (Figure 5A-B), morphants had a statistically significant increase of Sox $10^{+}$cells in the mandibular jaw joint region (Figure $5 \mathrm{C}-\mathrm{C}^{\prime}$ ). Consistent with these results, qPCR detected an increase in sox 10 expression at $3 \mathrm{dpf}$ (Figure 5D). However, at $4 \mathrm{dpf}$ (Figure 6A-B), the number of Sox $10^{+}$cells were reduced relative to control injected embryos (Figure $6 \mathrm{C}-\mathrm{C}^{\prime}$ ) and the level of sox 10 expressino was approximately $50 \%$ of the control according to qPCR (Figure 6D). Subsequent analysis of the number of Col2a1a (collagen, type II, alpha $1 \mathrm{a})^{+}$cells using the $\operatorname{Tg}$ (col2a1a:EGFP) transgenic reporter (Figure 7A-B) demonstrated a similar decline in numbers of EGFP+ cells in morphant animals at $4 \mathrm{dpf}$ (Figure $\left.7 \mathrm{C}-\mathrm{C}^{\prime}\right)$. Collectively, these data show a progressive loss of differentiated NCCs between 3 and $4 \mathrm{dpf}$.

\section{Discussion}


Our analysis revealed a $7 \%$ mandibular truncation and mandibular joint fusions in animals with reduced expression of $h s p g 2$. Mutations of Hspg2 in mice cause truncated snouts and mandibles $(3,6)$, but very little has been reported on the joint fusion phenotype. Although it is relatively novel, the idea of $h s p g 2$ mediating the mandibular jaw joint region is not completely unfounded. Similar to all other diarthrodial joints, the temporomandibular joint contains a synovial capsule, which, in previous cell culture work, has been shown to have $h s p g 2$ (perlecan) expression $(21,22)$. We further demonstrate that knockdown of $h s p g 2$ is associated with decreased numbers of Col2a $1 \mathrm{a}^{+}$cells at $4 \mathrm{dpf}$. These data suggest that $h s p g 2$ has a function regulating CNCC differentiation, a finding that is supported by the number of Sox $10^{+}$cells at an equivalent time point. Our studies are supported by previous analysis in mice $\left(\mathrm{Hspg}^{-/}\right)$that demonstrated abnormal arrangement and proliferation of chondrocytes in the appendicular skeleton (27). It must be noted however, that although these data support one another, the cells of the appendicular skeleton derive from a different germ layer (the mesoderm) than the cells of the craniofacial skeleton (the ectoderm) (22). Both cell lineages give rise to cartilaginous structures but the mechanisms by which each population differentiates are likely to differ between skeletal and craniofacial development, prompting further studies. Interestingly, we also observed an initial increase in the number of Sox $10+$ cells at $3 \mathrm{dpf}$, which at the onset seems to counter the results observed at $4 \mathrm{dpf}$. However, these differences might be explained by reduced cell proliferation or increased cell death between 3 and $4 \mathrm{dpf}$. Future studies in this area are warranted.

Knockdown of $h s p g 2$ was associated with reduced expression of $n k x 3.2$ at $4 \mathrm{dpf}$. These results, when understood in the context of the decrease of Col2a1 $\mathrm{a}^{+}$cells found at $4 \mathrm{dpf}$, appear to match previous results performed in mesenchymal cell culture where $n k x 3.2$ upregulates col2a 1 by directly binding to the promoter (23). In this situation, diminished expression of $n k x 3.2$ appears to be directly proportional to a decrease in Col2a1a+ cells and the differentiation of chondrocytes. However, it is not clear if hspg2 directly modulates $n k \times 3.2$ expression or if the decreased expression is simply the result of defects in mandibular jaw joint fusion.

In this paper, we demonstrate zebrafish as an alternative animal model to study the role of $h s p g 2$ during craniofacial development. Induced knockouts in the murine model have resulted in embryonic lethality through mass hemorrhaging in the pericardial cavity and severe chondrodysplasia, both occurrences which can be temporarily circumvented in the developing zebrafish (2). To circumvent these limitations, additional mouse models have been produced. However, these models restore early lethality via the tissue specific expression of $\mathrm{Hspg} 2$ in chondrocytes. Consequently, they cannot be utilized to study chondrogenesis or craniofacial development (24). Zebrafish additionally offer technical advantages. First, zebrafish are externally fertilized, enabling the study of craniofacial development in real time with transgenic reporter animals that currently exist (25). Secondly, facial development has been well characterized in zebrafish.

All the work reported here has been completed by use of a single translation-blocking morpholino. While translation-blocking morpholinos are a simple and effective way in which to knockdown genes of interest, they have been associated with off target effects and non-specific cell death. We did, however, utilize a 
random control morpholino to account for the possibility of morpholino-induced cell death, an endeavor that proved to be rather successful (experiments performed did not show a discrepancy between the noninjected wildtype and random control groups). Unfortunately, due to the size and availability of any perlecan encoding open reading frames, we were unable to perform rescue experiments. These are some potential caveats to our work. However, our data with one morpholino is supported by previous studies, including those completed using the murine model $(3,6)$. In these studies, numbers of chondrocytes in the lateral skeleton are depleted, chondrocytes congregate abnormally, and mutant mice exhibit craniofacial abnormalities. Our work contributes another angle to the role of $h s p g 2$ in skeletal development by examining the mandibular jaw joint region specifically. We understand that while additional morpholinos would help to substantiate our work, our data is supported by previous studies, suggesting that what we observed is not a consequence of off-target effects. Nevertheless, future studies with additional morpholino targets or a viable mutant allele are warranted.

\section{Conclusion}

In summary, our results have created novel implications for the role of $h s p g 2$ in the development of the mandibular jaw joint, a region of the craniofacial skeleton for which development has not been well elucidated. Furthermore, connections between $h s p g 2$ and $n k \times 3.2$ have yet to be drawn in literature. Additional data concerning late $\mathrm{CNCC}$ differentiation raises pertinent questions about the fate of certain cells within different areas of the developing face. Although future studies to more cohesively understand the role of $h s p g 2$ in craniofacial development are needed, these data lay significant groundwork for future experiments in this field and suggest that zebrafish are an acceptable model to study the function $h s p g 2$ in craniofacial development.

\section{Methods}

\section{Animal Care}

For all experiments, embryos were obtained by crossing adult $T g$ (sox10:tagRFP), $\operatorname{Tg}($ col2a1a:EGFP), and $A B$ wildtype fish. Embryos were maintained in $\mathrm{E} 3$ embryo medium at $28^{\circ} \mathrm{C}$. All zebrafish were maintained at The University of Texas El Paso according to the Institutional Animal Care and Use Committee (IACUC) guidelines protocol 811689-5. All adult larvae were obtained from the University of Colorado, Anschutz Medical Campus or the Zebrafish International Resource Center (ZIRC). Adult and larval zebrafish were euthanized and anesthetized according to guidelines from the American Veterinary Medical Association and approved IACUC protocols. For euthanasia, adults beyond the age of peak breeding age ( $>1.5$ years old) were euthanized using a solution of $10 \mathrm{~g} / \mathrm{L}$ buffered solution of pharmaceutical grade MS 222 . Fish were emerged in solution for 30 minutes at RT. All euthanized adults underwent secondary euthanasia with a cold ice bath (2-4 degrees $\mathrm{C}$ ). Cessation of movement was indicative of euthanasia. Embryos ( $<7$ days old) were euthanized using 1-10\% sodium hypochlorite solution after being anesthetized in cold ice bath. For any genotyping and before fixation, all fish, adults and larvae, were anesthetized using MS 222 
( $150 \mathrm{mg} / \mathrm{L}$ for adults and $300 \mathrm{mg} / \mathrm{L}$ for embryos). The degree of anesthesia was monitored by operculum movement of adults and cessation of movement for larvae.

\section{Antisense oligonucleotide morpholino design and microinjection}

Two antisense oligonucleotide morpholino sequences were designed in conjugation with Gene Tool LLC. The first was a translation blocking morpholino (MO) with the sequence $5^{\prime}$ -

TATCCTCGCCCCCATTTCTGCCAA-3', created to bind to the $h s p g 2$ translation start site and sterically knockdown perlecan translation in the developing larvae. The second was a random control morpholino with the sequence 5'-AAAAAAAAAAAAAAAAAAAAAAAA-3', used to assure that the MO microinjections were not causing any form of cell death as described by previous literature $(26,27)$. For all experiments, 3 experimental groups (morphants, random control, and wildtype) were injected (wildtype were noninjected) and compared. Both MOs were injected into embryos at the one cell stage with a stock concentration of $0.10 \mu \mathrm{M}$ and at a volume of $0.5 \mathrm{~nL}$ per embryo.

\section{Alcian Blue Staining and Imaging}

Zebrafish larvae (aged 5 days post fertilization (dpf)) were fixed in 2\% PFA in PBS, pH 7.5 for 1 hour at room temperature (RT). Samples were washed for 10 minutes with $100 \mathrm{mM}$ Tris $\mathrm{pH} 7.5 / 10 \mathrm{mM} \mathrm{MgCl2}$, stained with Alcian blue stain (pH 7.5: 0.4\% Alcian blue (Anatech Ltd., MI) in 70\% EtOH, Tris pH 7.5 (Fisher, MA), and $1 \mathrm{M} \mathrm{MgCl} 2$ (Fisher, MA)), and incubated overnight at RT. Samples were subsequently destained and rehydrated using an EtOH: Tris pH 7.5 gradient as previously described (28). Embryos were bleached (30\% H2O2 (Sigma, St. Louis, MO), 20\% KOH (Fisher, MA)) for 10 minutes at RT. Samples were washed twice for 10 minutes per wash in wash buffer $(25 \%$ glycerol $/ 0.1 \% \mathrm{KOH}$ (Fisher, MA)) and stored at $4^{\circ} \mathrm{C}$ in storage buffer ( $50 \%$ glycerol $/ 0.1 \% \mathrm{KOH}$ (Fisher, MA)) until imaged. The distance between the Meckel's cartilage (an intermediate cartilaginous structure, which makes up the ventral component of the mandibular arch) (29) and the ceratohyal (a pharyngeal arch cartilage) (30) was measured for each embryo as previously described (17).

For imaging, a representative sample of each group ( $h s p g 2$ morphants, random control, and non-injected wildtype) was dissected and the viscerocranium (the mandibular jaw region) was mounted on a glass slide with $100 \%$ glycerol. A Leica microscope was used to take high-resolution color images of each sample.

\section{Whole mount in situ hybridization and quantitative real time polymerase chain reaction (PCR)}

Whole mount in situ hybridization was performed as described by Thisse and Thisse (31). Larvae were harvested and dechorionated at the indicated time point and fixed in $4 \%$ paraformaldehyde (Electron Microscopy Sciences, PA) overnight at $4^{\circ} \mathrm{C}$. Larvae were then dehydrated using a methanol: PBS gradient and stored in $100 \%$ methanol overnight at $-20^{\circ} \mathrm{C}$. Embryos were rehydrated using a PBS:Methanol gradient, washed in PBS with $0.1 \%$ Tween 20 and permeabilized with proteinase $\mathrm{K}(10 \mathrm{ug} / \mathrm{ml})$ for the time indicated by Thisse and Thisse (31). Permeabilized larvae were prehybridized for 2 hours in hybridization 
buffer (HB) (50\% deionized formamide (Fisher), 5X SSC (Fisher), 0.1\% Tween 20 (Fisher), $50 \mu \mathrm{g} / \mathrm{m}$ heparin (Sigma), $500 \mu \mathrm{g} / \mathrm{mL}$ of RNase-free tRNA (Sigma), and $1 \mathrm{M}$ citric acid (Fisher). Larvae were then incubated overnight in fresh HB with probe ( $d 1 \times 2 a$ and $n k \times 3.2$ at $127 \mathrm{ng})$ at $70^{\circ} \mathrm{C}$. Samples were washed according to protocol, blocked in $2 \%$ sheep serum (Sigma) and $2 \mathrm{mg} / \mathrm{ml}$ bovine albumin serum (Sigma) for 2 hours at room temperature. Samples were then incubated with anti-DIG Fab fragments $(1: 10,000)$ (Sigma) overnight at $4^{\circ} \mathrm{C}$. Samples were developed with BM purple AP substrate (Sigma) and imaged with a Zeiss Discovery Stereo Microscope fitted with Zen Software. Statistical analysis was performed using a Fisher's exact test. For quantitative polymerase chain reaction (qPCR), RNA was isolated from embryos at the indicated time point using Trizol (Fisher) according to manufacturer's protocol. Reverse transcription was performed using a Verso cDNA Synthesis Kit (Fisher) and total RNA was normalized across all samples. PCR was performed using an Applied Biosystem's StepOne Plus machine with Applied Biosystem's software. Sybr green (Fisher) based primer pairs for each gene analyzed are as follows: $d l x 2 a$ fwd CCTCACGCAAACACAGGTTA, $d$ l $x 2 a$ rev TGTTCATTCTCTGGCTGTGC, $n k x 3.2 \mathrm{fwd}$ GCAGATTTAGCGGACGAGAC, $n k x 3.2$ rev GCTTCAACCACCAGCGTTAT, sox10 fwd ACGCTACAGGTCAGAGTCAC, sox10 rev ATGTTGGCCATCACGTCATG, rp/13a fwd TCCCAGCTGCTCTCAAGATT, and rp/13a rev TTCTTGGAATAGCGCAGCTT. Analysis performed using $2^{\triangle \triangle c t}$. Statistical analysis of messenger RNA (mRNA) expression was performed using a Student $t$ test on biological replicates. All qPCR was performed in biological duplicates.

\section{Confocal Imaging and Transgenic Cell Counts}

Transgenic larvae ( $\mathrm{Tg}$ (sox10:tagRFP) and $\mathrm{Tg}$ (col2a1a:EGFP)) were fixed at given time points using 4\% paraformaldehyde. Fixed larvae were mounted in $0.6 \%$ low-melt agar in a glass bottom dish (Fisher). Imaging was performed on a Zeiss LSM 700 at 20X and 40X Oil magnification. Images were restricted to the larval craniofacial region. For each fish, a minimum of 20 to $30 \mathrm{z}$-stacks were collected. Maximum intensity projection images were processed and saved (32). For cell counts, the number of cells per zstack (20-30/fish) at each jaw joint region (3 rows of chondrocytes on the left side of the joint and 5 rows on the right) were counted using ImageJ. Statistical significance was obtained using a Student $t$ test (32).

\section{Declarations}

\section{Ethics approval and consent to participate}

All experiments were performed according to protocol 811689-5 approved by The University of Texas El Paso Institutional Animal Care and Use Committee (IACUC).

\section{Consent for publication}

Not applicable.

\section{Availability of data and materials}


Not applicable.

\section{Competing interests}

The authors declare that they have no competing interests.

\section{Funding}

National Institute of Neurological Disorders and Stroke 1K01NS099153-01A1 to Anita M. Quintana. This study was designed, performed, and analyzed by the authors. The funding sources provided financial support for the experiments described.

\section{Authors' contributions}

BSC and AMQ synthesized hypothesis, wrote manuscript and BSC performed all experiments described. All authors included have read and approved the manuscript being submitted.

\section{Acknowledgments}

We would like to acknowledge the rest of the Quintana lab members for their help and support in animal care, aquarium maintenance, and in offering pertinent critical feedback for this project throughout its development.

\section{Abbreviations}

HSPG2/Hspg2/hspg2: heparan sulfate proteoglycan 2; dpf: days-post-fertilization, NCC: neural crest cells; sox 10: SRY-box transcription factor 10; $n k x 3.2$ : NK3 homeobox 2; SJS: Schwartz-Jampel Syndrome; DDSH: Dyssegmental Dysplasia Handmaker Type; qPCR: quantitative polymerase chain reaction; col2a1a: collagen, type II, alpha 1a; MO: hspg2 morphants; NI: non-injected wildtype; RC: random control group

\section{References}

1. Reference GH. HSPG2 gene [Internet]. Genetics Home Reference. [cited 2019 Oct 15]. Available from: https://ghr.nlm.nih.gov/gene/HSPG2

2. Zoeller JJ, McQuillan A, Whitelock J, Ho S-Y, lozzo RV. A central function for perlecan in skeletal muscle and cardiovascular development. J Cell Biol. 2008 Apr 21;181(2):381-94.

3. Arikawa-Hirasawa E, Watanabe $H$, Takami H, Hassell JR, Yamada Y. Perlecan is essential for cartilage and cephalic development. Nat Genet. 1999 Nov;23(3):354-8.

4. Nicole S, Davoine CS, Topaloglu H, Cattolico L, Barral D, Beighton P, et al. Perlecan, the major proteoglycan of basement membranes, is altered in patients with Schwartz-Jampel syndrome (chondrodystrophic myotonia). Nat Genet. 2000 Dec;26(4):480-3. 
5. Arikawa-Hirasawa E, Le AH, Nishino I, Nonaka I, Ho NC, Francomano CA, et al. Structural and functional mutations of the perlecan gene cause Schwartz-Jampel syndrome, with myotonic myopathy and chondrodysplasia. Am J Hum Genet. 2002 May;70(5):1368-75.

6. Rodgers KD, Sasaki T, Aszodi A, Jacenko O. Reduced perlecan in mice results in chondrodysplasia resembling Schwartz-Jampel syndrome. Hum Mol Genet. 2007 Mar 1;16(5):515-28.

7. Stum M, Davoine C-S, Vicart S, Guillot-Noël L, Topaloglu H, Carod-Artal FJ, et al. Spectrum of HSPG2 (Perlecan) mutations in patients with Schwartz-Jampel syndrome. Hum Mutat. 2006;27(11):108291.

8. Micrognathia | Children's Hospital of Philadelphia [Internet]. [cited 2019 Sep 23]. Available from: https://www.chop.edu/conditions-diseases/micrognathia

9. Schwartz Jampel syndrome | Genetic and Rare Diseases Information Center (GARD) - an NCATS Program [Internet]. [cited 2019 Sep 23]. Available from: https://rarediseases.info.nih.gov/diseases/250/schwartz-jampel-syndrome

10. Dyssegmental dysplasia Silverman-Handmaker type | Genetic and Rare Diseases Information Center (GARD) - an NCATS Program [Internet]. [cited 2019 Sep 30]. Available from: https://rarediseases.info.nih.gov/diseases/2026/dyssegmental-dysplasia-silverman-handmaker-type

11. Chondrodysplasia - an overview | ScienceDirect Topics [Internet]. [cited 2019 Oct 17]. Available from: https://www.sciencedirect.com/topics/medicine-and-dentistry/chondrodysplasia

12. Chondrocyte - an overview | ScienceDirect Topics [Internet]. [cited 2019 Sep 17]. Available from: https://www.sciencedirect.com/topics/biochemistry-genetics-and-molecular-biology/chondrocyte

13. Signals and Switches in Mammalian Neural Crest Cell Differentiation [Internet]. [cited 2019 Oct 18]. Available from: https://www.ncbi.nlm.nih.gov/pmc/articles/PMC3552505/

14. Cordero DR, Brugmann S, Chu Y, Bajpai R, Jame M, Helms JA. Cranial neural crest cells on the move: their roles in craniofacial development. Am J Med Genet A. 2011 Feb;155A(2):270-9.

15. Zhang D, Ighaniyan S, Stathopoulos L, Rollo B, Landman K, Hutson J, et al. The neural crest: A versatile organ system. Birth Defects Res Part C Embryo Today Rev. 2014;102(3):275-98.

16. Lukas P, Olsson L. Bapx1 is required for jaw joint development in amphibians. Evol Dev. 2018;20(6):192-206.

17. Quintana AM, Hernandez JA, Gonzalez CG. Functional analysis of the zebrafish ortholog of HMGCS1 reveals independent functions for cholesterol and isoprenoids in craniofacial development. PloS One. 2017;12(7):e0180856.

18. Klymkowsky MW, Rossi CC, Artinger KB. Mechanisms driving neural crest induction and migration in the zebrafish and Xenopus laevis. Cell Adhes Migr. 2010;4(4):595-608.

19. Zebrafish Zic2a and Zic2b regulate neural crest and craniofacial development. - PubMed - NCBI [Internet]. [cited 2019 Oct 20]. Available from: https://www.ncbi.nlm.nih.gov/pubmed/23665173

20. LaMonica K, Ding H, Artinger KB. prdm1 a functions upstream of itga5 in zebrafish craniofacial development. Genes N Y N 2000. 2015 Apr;53(3-4):270-7. 
21. Lowe DA, Srinivasan PP, Kirn-Safran C. Chondrocyte Phenotype of Mice Expressing Reduced Levels of Perlecan/HSPG2, an Essential Component of Cartilage. FASEB J. 2012 Apr 1;26(1_supplement):971.6-971.6.

22. Development of the Appendicular Skeleton | Anatomy and Physiology I [Internet]. [cited 2019 Oct 29]. Available from: https://courses.lumenlearning.com/suny-ap1/chapter/development-of-theappendicular-skeleton/

23. Kawato Y, Hirao M, Ebina K, Shi K, Hashimoto J, Honjo Y, et al. Nkx3.2 promotes primary chondrogenic differentiation by upregulating Col2a1 transcription. PloS One. 2012;7(4):e34703.

24. Perlecan, a heparan sulfate proteoglycan, regulates systemic metabolism with dynamic changes in adipose tissue and skeletal muscle | Scientific Reports [Internet]. [cited 2019 Sep 30]. Available from: https://www.nature.com/articles/s41598-018-25635-x\#Sec9

25. Allen JP, Neely MN. Trolling for the ideal model host: zebrafish take the bait. Future Microbiol. 2010 Apr;5(4):563-9.

26. Heasman J. Morpholino oligos: making sense of antisense? Dev Biol. 2002 Mar 15;243(2):209-14.

27. Reyes-Nava N, Yu H-C, Coughlin CR, Shaikh TH, Quintana AM. Abnormal expression of GABAA receptor sub-units and hypomotility upon loss of gabra1 in zebrafish. Biol Open [Internet]. 2020 Jan 1 [cited 2020 Apr 9]; Available from: https://bio.biologists.org/content/early/2020/03/20/bio.051367

28. Quintana AM, Geiger EA, Achilly N, Rosenblatt DS, Maclean KN, Stabler SP, et al. Hcfc1b, a zebrafish ortholog of HCFC1, regulates craniofacial development by modulating mmachc expression. Dev Biol. 2014 Dec 1;396(1):94-106.

29. Meckel's Cartilage - an overview / ScienceDirect Topics [Internet]. [cited 2019 Sep 23]. Available from: https://www.sciencedirect.com/topics/veterinary-science-and-veterinary-medicine/meckels-cartilage

30. ZFIN Anatomy Ontology: ceratohyal cartilage [Internet]. [cited 2019 Sep 23]. Available from: https://zfin.org/ZFA:0001400

31. Thisse C, Thisse B. High-resolution in situ hybridization to whole-mount zebrafish embryos. Nat Protoc. 2008;3(1):59-69.

32. Hernandez JA, Castro VL, Reyes-Nava N, Montes LP, Quintana AM. Mutations in the zebrafish hmgcs 1 gene reveal a novel function for isoprenoids during red blood cell development. Blood Adv. 2019 23;3(8):1244-54.

\section{Figures}


A.

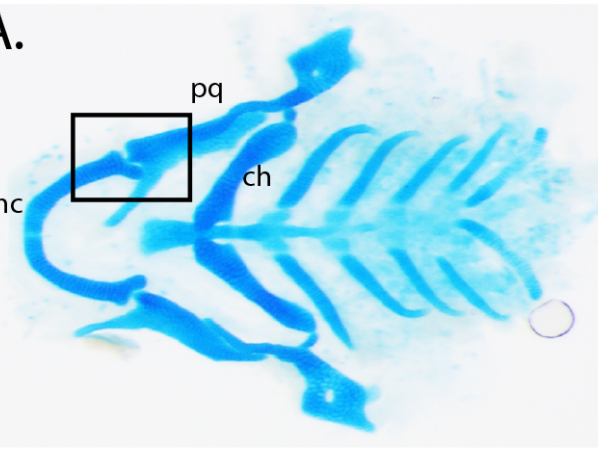

$A^{\prime} \cdot$

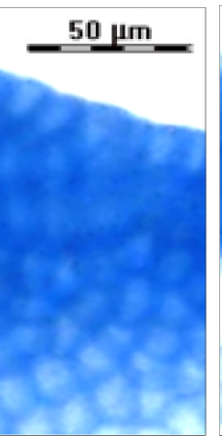

B.

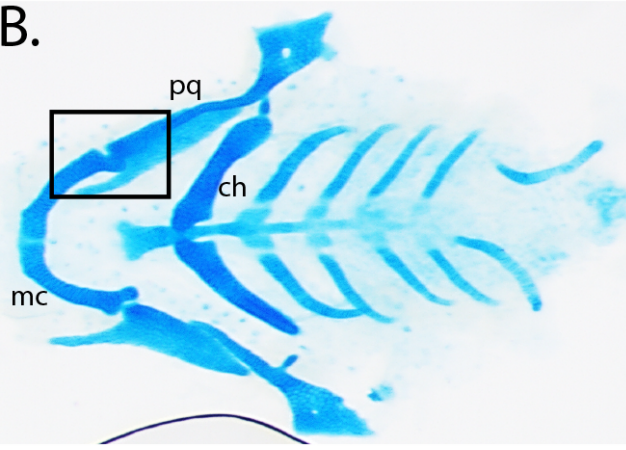

C.
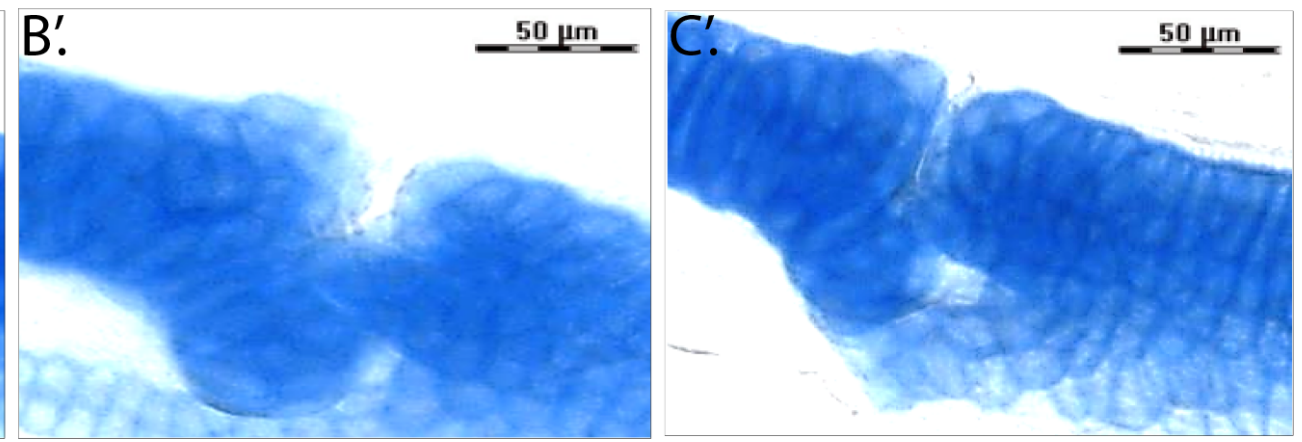

D.

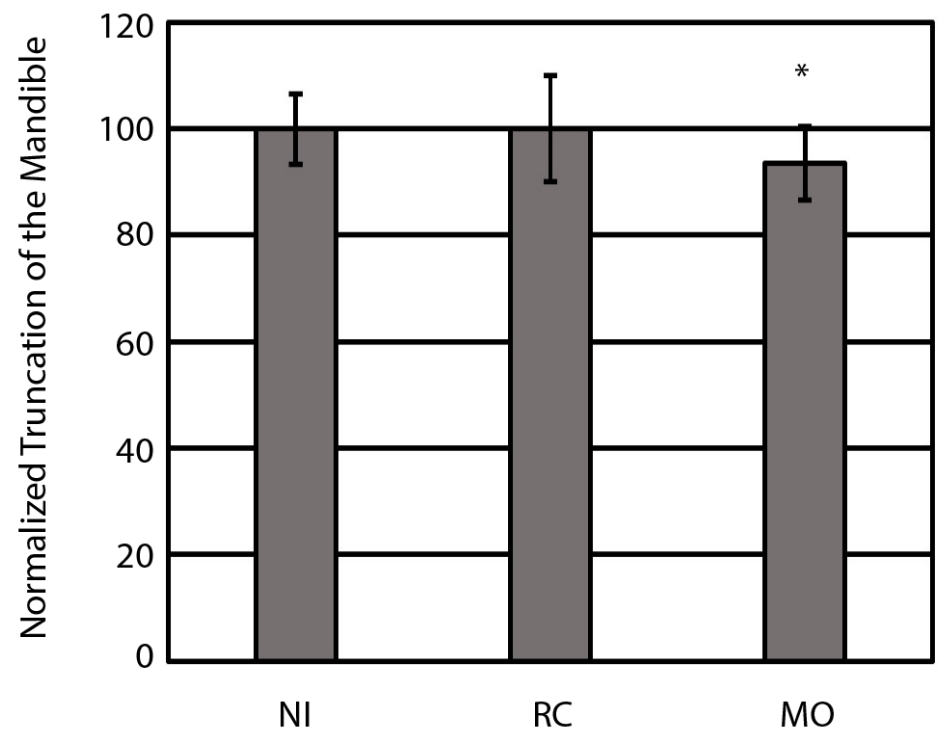

Figure 1

Knockdown of hspg2 is associated with mandibular jaw joint fusion. (A-C) Non-injected wildtype (NI), random control (RC), and morphant (MO) groups were stained with Alcian blue at 5 days post fertilization (dpf) ( $\mathrm{N}=20$ per group). Ceratohyal, Meckel's cartilage, and the palatoquadrate are labeled as the abbreviations (ch), (mc), and (pq) respectively. The black box on each of the images shows the upper jaw joint. ( $\left.A^{\prime}-C^{\prime}\right)$ shows the upper mandibular jaw joint enclosed by the black box at $40 \mathrm{X}$ magnification. Images collectively show a fusion in the mandibular jaw joint of the morphants (C'). (D) The distance 
between the Meckel's cartilage and ceratohyal was measured across all three groups ( $N=20$ per group). Mandibular length was normalized to the random control (RC) group. * $P=0.025$.

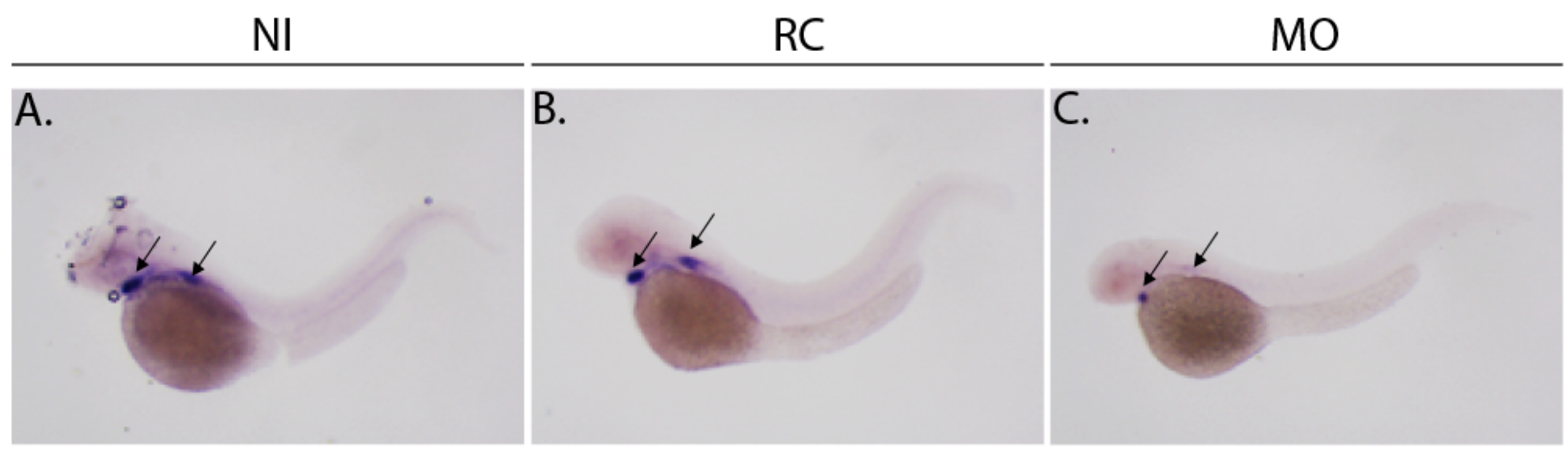

D.

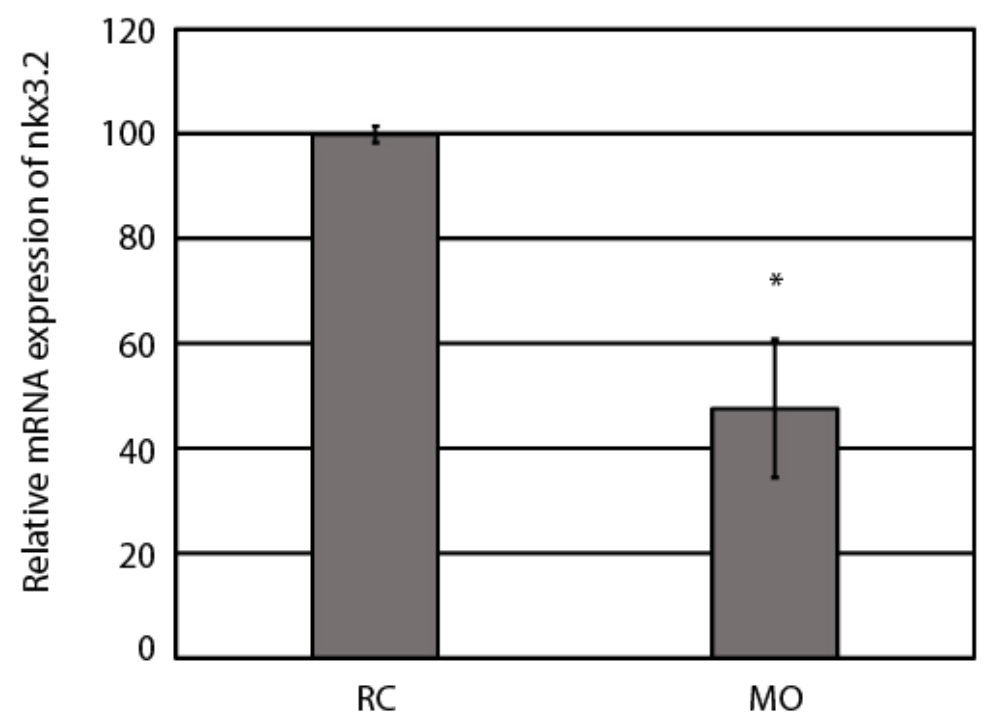

Figure 2

nkx3.2 expression is decreased in hspg2 morphants (A-C) Whole mount in situ hybridization (ISH) was performed to detect the expression of the $\mathrm{nkx} 3.2$ at the 2 days post fertilization (dpf) stage. Embryos were injected as described in methods section and subjected to ISH to detect nkx3.2 expression in the developing jaw joint region. Black arrows dictate the expression of nkx3.2. $\mathrm{N}=14$ larvae the non-injected group (NI), $N=18$ in random control (RC) group, and $N=14$ larvae in hspg2 morphants (MO) group. (D) qPCR was performed to detect the expression of nkx3.2 at $4 \mathrm{dpf}$. RC and hspg2 MO samples ( $\mathrm{N}=15$ total per group); error bars represent the standard deviation of biological replicates ( $\left.{ }^{*} \mathrm{P}=0.004\right)$. 

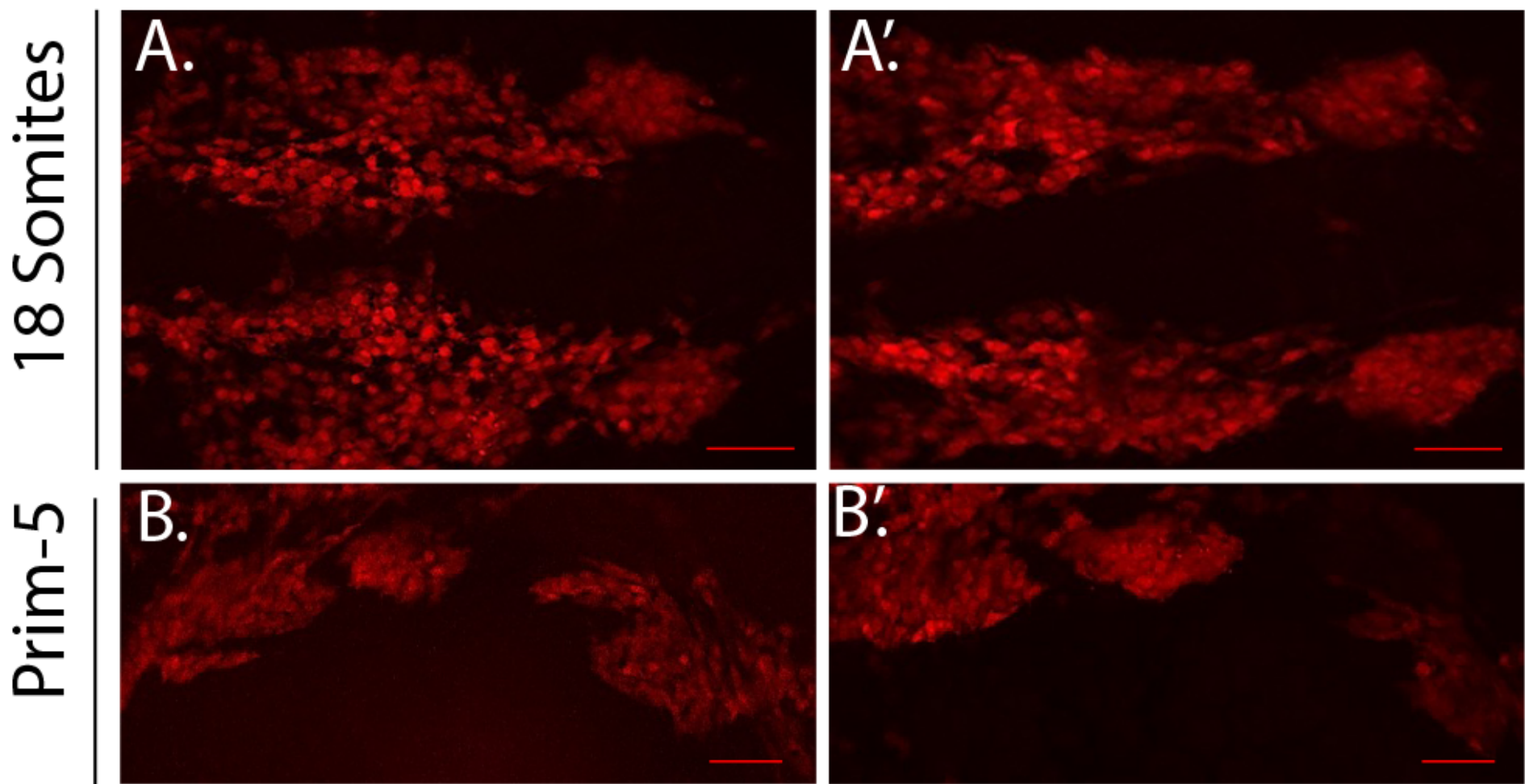

\section{Figure 3}

Early neural crest cell (NCC) migration and specification are normal in morphants. (A-A') Non-injected (NI) $\mathrm{Tg}$ (sox10:TagRFP) larvae and hspg2 morpholino injected Tg(sox10:TagRFP) larvae (MO) ( $N=6$ and $N=4$, respectively) larvae were staged and fixed at the 18-somite stage. Images are scaled at $200 \mu \mathrm{m}$ and demonstrate two lateral streams of Sox $10+$ migrating NCCs. (B-B') $\mathrm{NI}$ and $\mathrm{MO}$ larvae $(\mathrm{N}=8$ and $\mathrm{N}=16$ respectively) were staged and fixed at the Prim-5 stage. Images are scaled at $200 \mu \mathrm{m}$ and demonstrate Sox $10+$ NCCs in the pharyngeal arches. No significant changes were found between the two groups at either timepoint. 

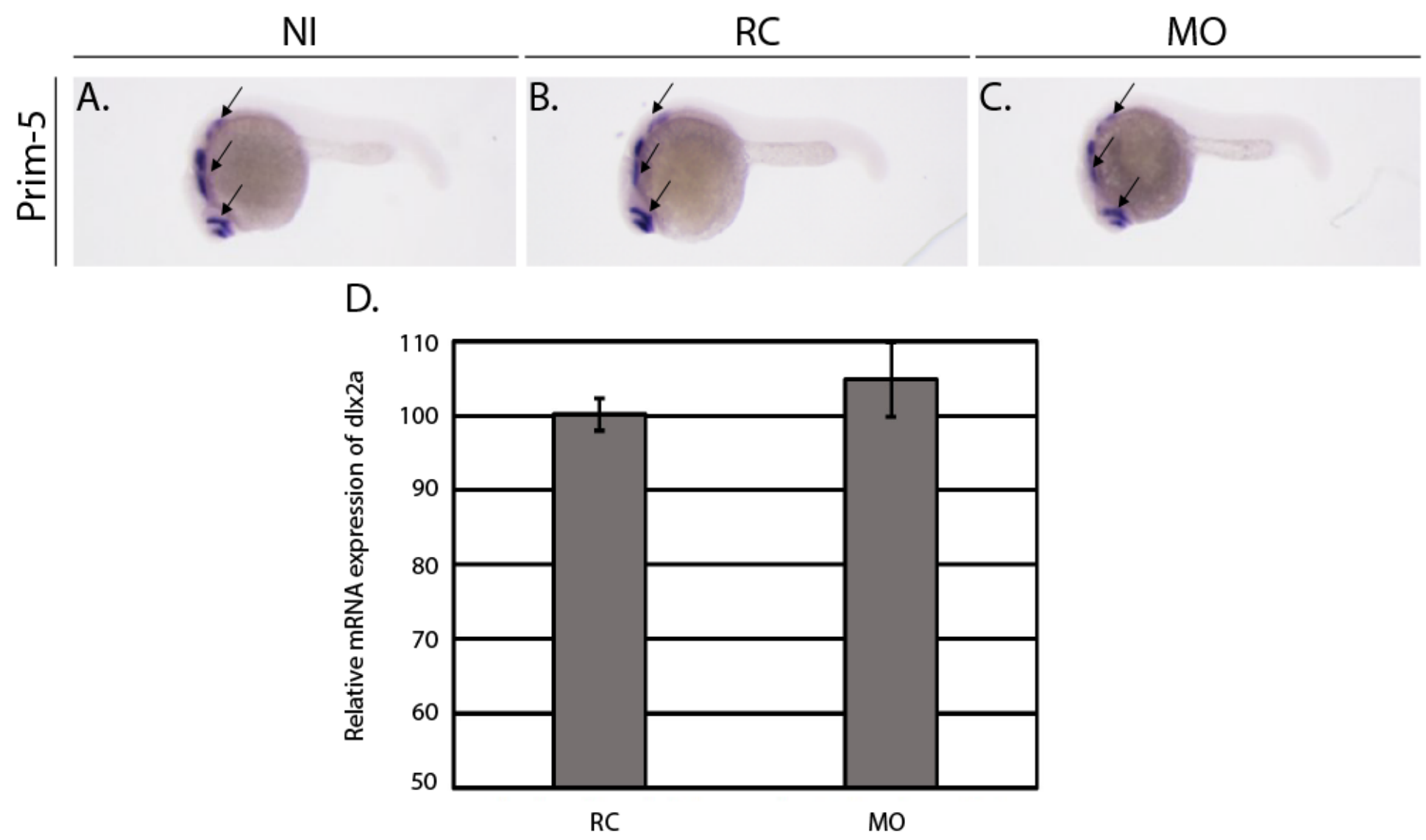

Figure 4

dlx2a expression is normal in morphants. (A-C) Whole mount in situ hybridization (ISH) was performed to detect the expression of the dlx2a at the Prim-5 stage ( $\mathrm{N}=10$ larvae for non-injected ( $\mathrm{NI})$ group, $\mathrm{N}=12$ larvae in random control (RC) group, and $\mathrm{N}=11$ larvae in hspg2 morphants (MO) group). Embryos were injected as described in the methods section and subjected to ISH to detect dlx2a expression in the pharyngeal arches labeled by black arrows. (D) qPCR was performed to detect the expression of dlx2a. Total RNA was isolated from RC and hspg2 MO samples ( $\mathrm{N}=10$ per group); error bars represent the standard deviation of biological replicates. Quantification between the two groups was not statistically significant. 

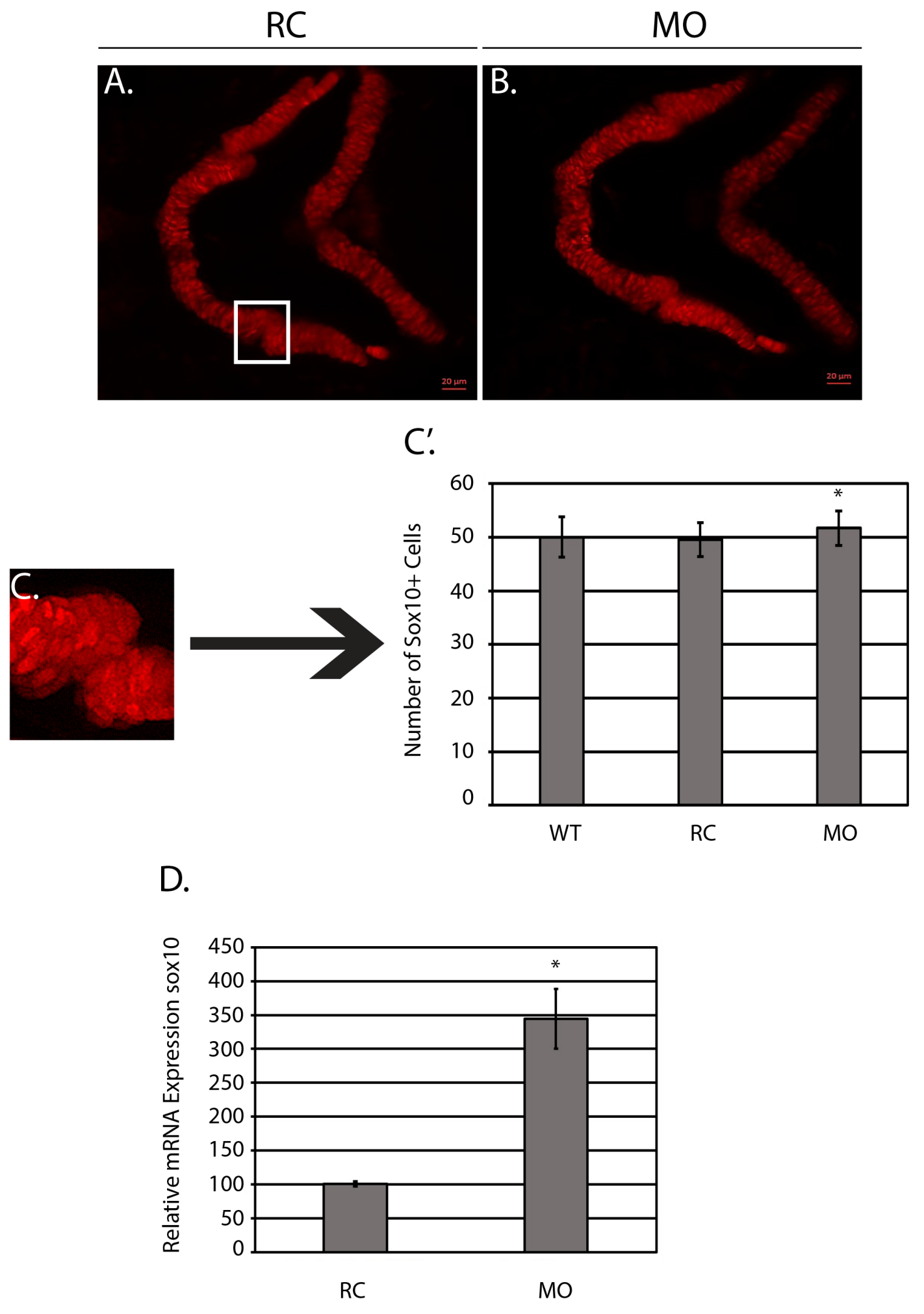

\section{Figure 5}

The number of Sox $10+$ cells is increased in morphants at 3 dpf. (A-B) $\operatorname{Tg}($ sox 10:TagRFP) random control $(\mathrm{RC})$ and morphant $(\mathrm{MO})$ larvae ( $\mathrm{N}=10$ per group) were mounted in agarose and confocal images were taken at 3 days post fertilization (dpf). Region surrounded by the white box (C) shows the region where cells were quantified (3 rows left, 5 rows right). ( $\left.C^{\prime}\right)$ Average number of Sox $10+$ cells counted across the three groups ( $\mathrm{N}=10$ per group) at $3 \mathrm{dpf}$. P-value pertains to the statistically significant difference between 
the RC group and the MO ( $\left.{ }^{*} \mathrm{P}=0.04\right)$. (D) $\mathrm{qPCR}$ expression of sox10 ( $\mathrm{N}=24$ total) in $\mathrm{RC}$ and $\mathrm{MO}$ groups at $3 \mathrm{dpf}\left({ }^{*} \mathrm{P}=0.0005\right)$. Error bars represent the standard deviation of biological replicates.

$\mathrm{RC}$

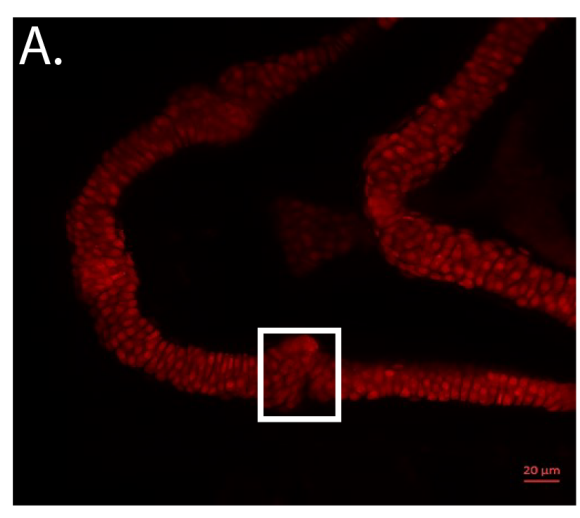

\section{$C^{\prime}$}
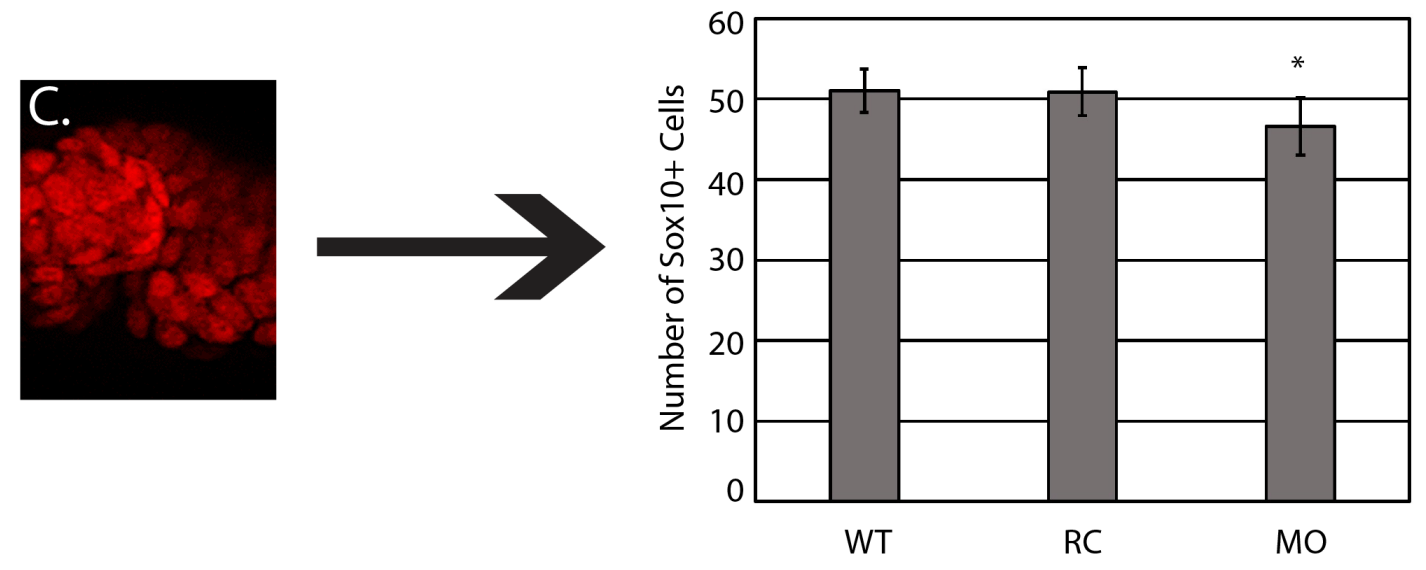

D.

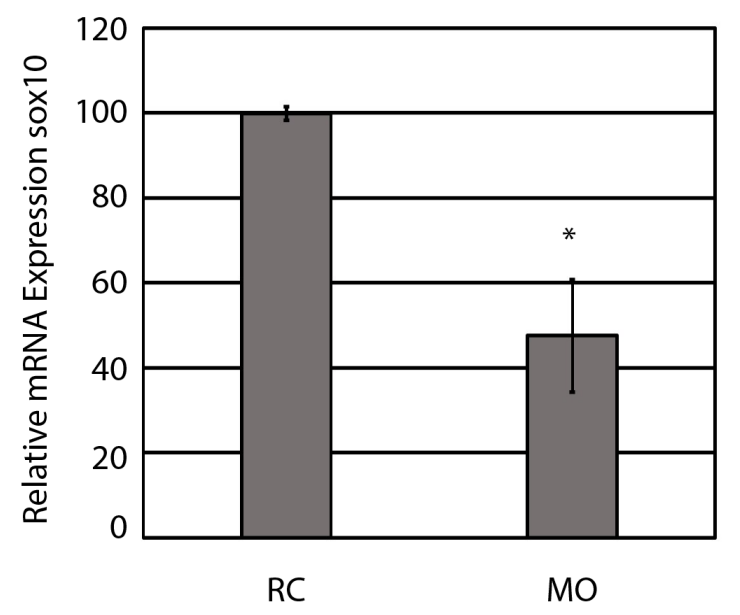

\section{Figure 6}

The number of Sox $10+$ cells is decreased in morphants at $4 \mathrm{dpf}$. (A-B) $\operatorname{Tg}(\operatorname{sox} 10: \operatorname{TagRFP})$ random control $(\mathrm{RC})$ and morphant $(\mathrm{MO})$ larvae ( $\mathrm{N}=10$ per group) were mounted in agarose and confocal images were taken at 4 days post fertilization (dpf). Region surrounded by the white box (C) shows the representative 
region of cells that was quantified ( 3 rows left, 5 rows right). ( $\left.C^{\prime}\right)$ Average number of Sox $10+$ cells counted across the three groups ( $N=10$ per group) at $4 \mathrm{dpf}$. P-value pertains to the statistically significant difference between the RC group and the MOs ( $\left.{ }^{*} P=0.0002\right)$. (D) $q P C R$ demonstrating the expression of sox10 ( $N=15$ total) in $R C$ and $M O$ groups at $4 \mathrm{dpf}\left({ }^{*} P=0.005\right)$. Error bars represent the standard deviation of biological replicates.
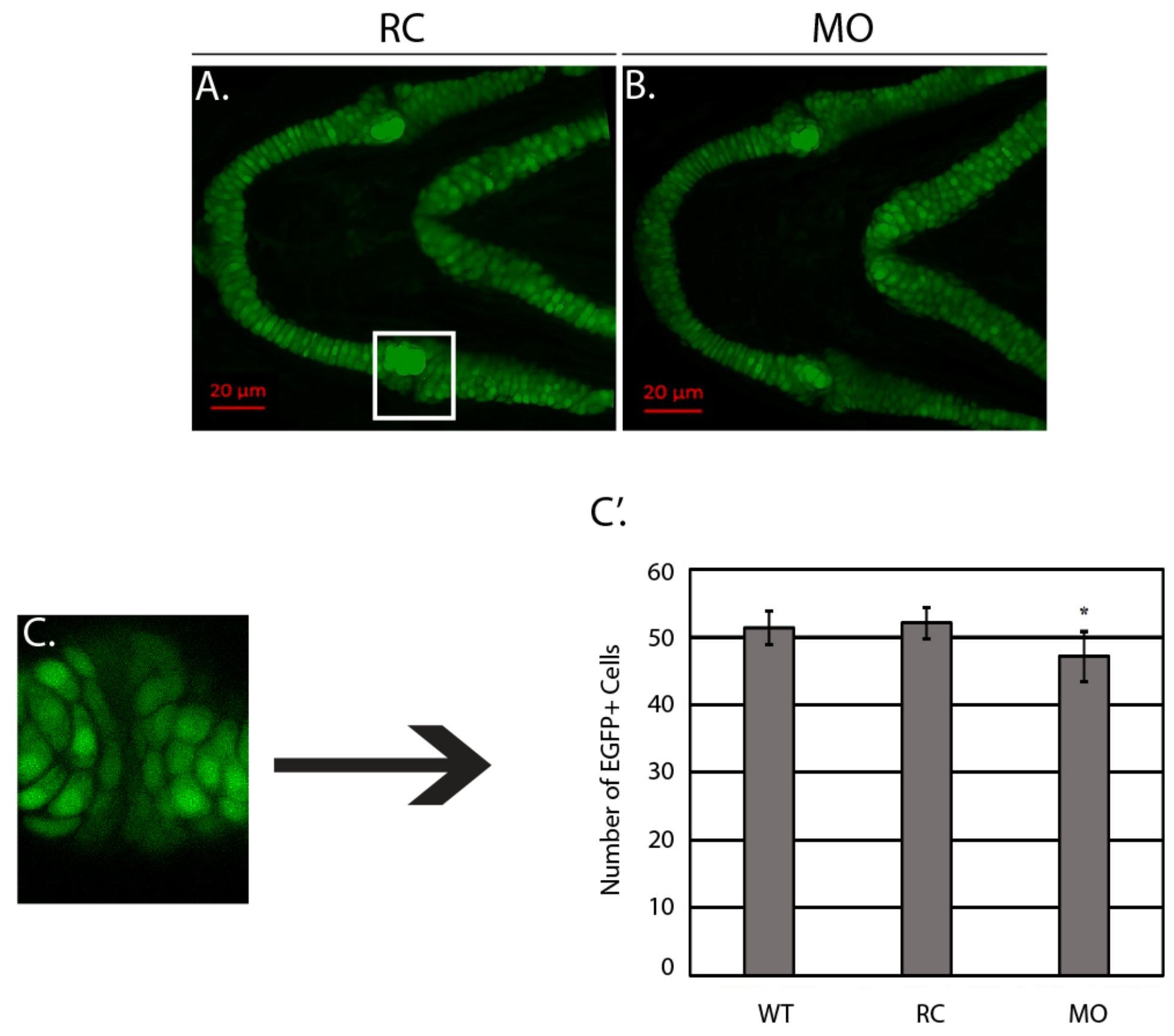

Figure 7

The number of Col2a1a+ cells is decreased at $4 \mathrm{dpf}$. (A-B) $\mathrm{Tg}$ (col2a2a:EGFP) random control (RC) and morphant (MO) larvae ( $\mathrm{N}=10$ per group) were mounted in agarose and confocal images were taken at 4 days post fertilization (dpf). Region surrounded by the white box (C) shows the representative region where cells were quantified (3 rows left, 5 rows right). (C') Average number of Col2a1a+ cells counted 
across the three groups ( $\mathrm{N}=10$ per groups) at $4 \mathrm{dpf}$. P-value pertains to the statistically significant difference between the RC group and the MOs ( $\left.{ }^{P}=1.44 x-05\right)$.

\section{Supplementary Files}

This is a list of supplementary files associated with this preprint. Click to download.

- NC3RsARRIVEGuidelinesChecklist.pdf 